$\mathrm{DE}$

M E D I C I N A

T R O P I C A L

$\mathrm{DE}$

SÃO PAULO

JOURNAL OF THE SÃO PAULO INSTITUTE OF TROPICAL MEDICINE

'Universidade de São Paulo, Faculdade de Medicina, Departamento de Dermatologia, São Paulo, São Paulo, Brazil

2Universidade de São Paulo, Faculdade de Medicina, Hospital das Clínicas, Laboratório de Investigação Médica (LIM-50), São Paulo, São Paulo, Brazil

${ }^{3}$ Faculdade de Medicina do ABC, Santo André, São Paulo, Brazil

Correspondence to: Walter Belda Junior Universidade de São Paulo, Faculdade de Medicina, Hospital das Clínicas, Laboratório de Investigação Médica (LIM-50), Av. Dr. Eneas de Carvalho Aguiar, 255, $3^{\circ}$ andar, CEP 05403-000, São Paulo, SP, Brazil Tel: +551126613391

E-mail: walterbelda@uol.com.br, walterbelda26@gmail.com

Received: $1^{\text {st }}$ March 2020

Accepted: 3 June 2020

\section{Atypical clinical presentation of an Arthroderma gypseum infection in a renal transplant recipient}

\author{
Walter Belda Junior (10) 1,2 , Paulo Ricardo Criado (1) 2,3
}

\section{ABSTRACT}

Dermatophytes are known as a common cause of superficial mycosis, but atypical presentations in immunosuppressed patients make the diagnosis more challenging. Here, we report a case of a 39-year-old patient, a renal transplant recipient from a living donor, who presented with atypical cutaneous lesions of lower extremities caused by Arthroderma gypseum (Nannizzia gypsea), four months after receiving a renal transplant. It is important to highlight the importance of the early detection of fungal infections in immunosuppressed patients. Clinicians should have a high degree of suspicion for the early detection and treatment of the cases.

KEYWORDS: Arthroderma gypseum. Dermatophytes. Transplanted patient.

\section{INTRODUCTION}

Superficial fungal infections caused by dermatophytes affect 20 to $25 \%$ of the world population. The predominant causative species vary geographically and commonly include Trichophyton, Arthroderma and Epidermophyton ssp. Fungal elements are generally restricted to the corneal layer of the epidermis, producing typical lesions with erythema and scaling.

However, in patients undergoing solid organ transplantation, skin lesions are frequent and due to the immunosuppression they are submitted to. Oliveira et al. ${ }^{1}$ evaluated 177 patients who underwent solid organ transplantation, having found 147 (83\%) with skin lesions, 107 (60\%) with infections, 30\% with common warts and 28 (16\%) with superficial mycoses, onychomycosis being the most frequent, followed by extensive scaling lesions in the inguinal region, abdomen and trunk in $9 \%$ of cases, the majority being caused by Trichophyton rubrum. Another study ${ }^{2}$ carried out on 223 patients undergoing organ transplantation, found 108 cases (46\%) of superficial fungal infections, with the oral cavity most frequently affected by Candida albicans, followed by mycosis of the nails, inguinal region, abdomen and trunk by Trichophyton $s p$.

However, in transplant recipients, atypical forms of fungal infection are described in the literature, underlining the need for a differential diagnosis with other diseases and even with deep mycosis ${ }^{3}$. Although atypical lesions caused by dermatophytes such as Trycophyton rubrum and Microsporum canis ${ }^{4,5}$ have already been reported in the literature, to the best of our knowledge ,there is no report on Arthroderma gypseum (Nannizzia gypsea) as a cause of deep mycosis in a renal transplant recipient, mimicking hyalohyphomycosis.

\section{CASE REPORT}

A 39-year-old female patient presented with pruritic erythematous papules 
on the right side of the ankle, four months after receiving a renal transplant. At this time, the patient was using azathioprine $150 \mathrm{mg}$ orally daily, cyclosporine $250 \mathrm{mg}$ orally twice a day and prednisone $40 \mathrm{mg}$ orally daily. The patient reported having a controlled high blood pressure and did not report a previous or present history of other comorbidities. The lesions increased in size and spread bilaterally to both lower extremities. Subsequently, they progressed to ulcerated plaques with a blood-draining $\mathrm{s}$ discharge (Figure 1).

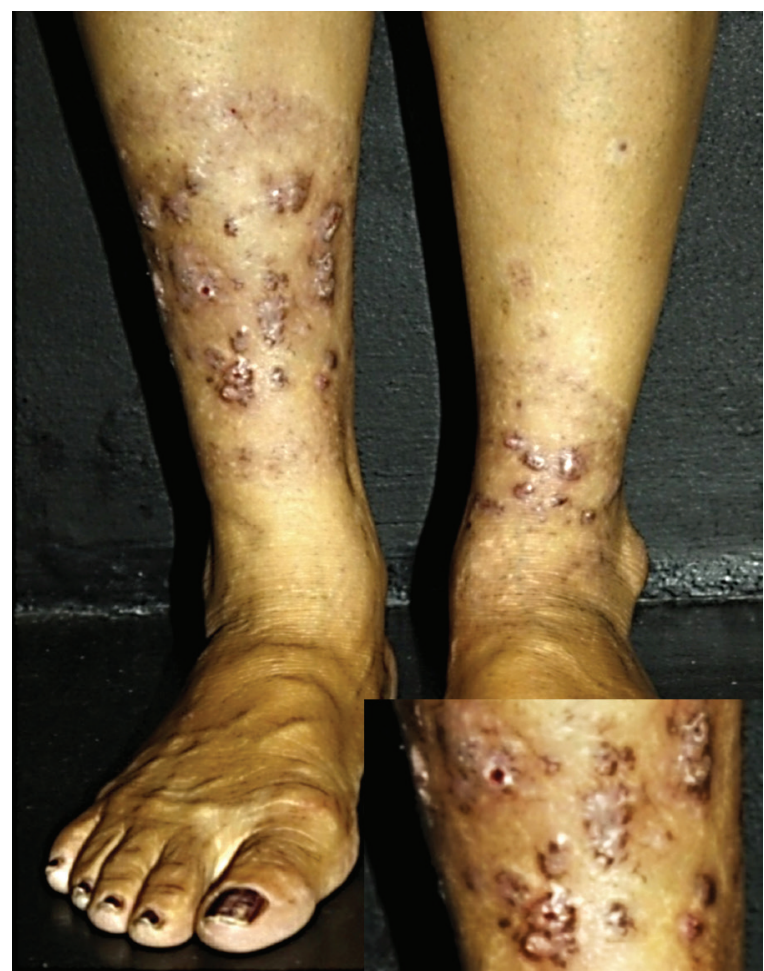

Figure 1 - Erythematous and violaceous papules and nodules, some of them with exulcerated surface on the limbs.

The confirmation of deep mycosis by $M$. gypseum ( $N$. gypsea) was made through a direct mycological examination, culture in Agar Sabouraud (Figure 2) and a cutaneous biopsy, however, the molecular identification of the strain was not performed. The histopathology demonstrated acanthosis and spongiosis of epidermis, a granulomatous and suppurative intense inflammation of the dermis and hypodermis (Figure 3) along with the presence of fungal septate hyphae in Grocott' staining (Figure 4).

The treatment with oral Itraconazole (100 mg per day) was carried out for two months, however, due to its potential kidney injury, it was replaced by terbinafine $(250 \mathrm{mg}$ per day) for more three months. The cutaneous lesions improved with progressive healing, eventually leading to multiple violaceous nodules on both lower extremities.

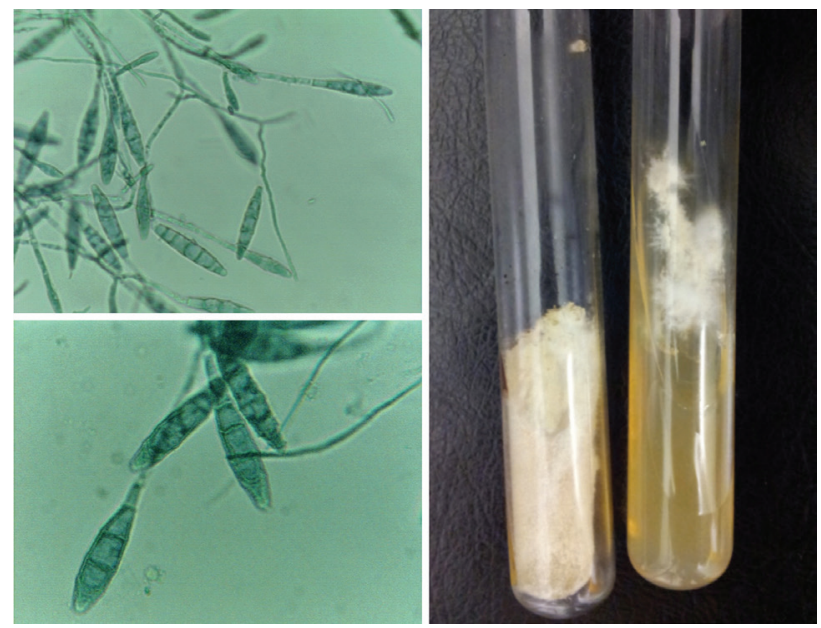

Figure 2 - Microculture showing macroconidia of Arthroderma gypseum and Macroculture on Agar Sabouraud displaying the Arthroderma gypseum growth.
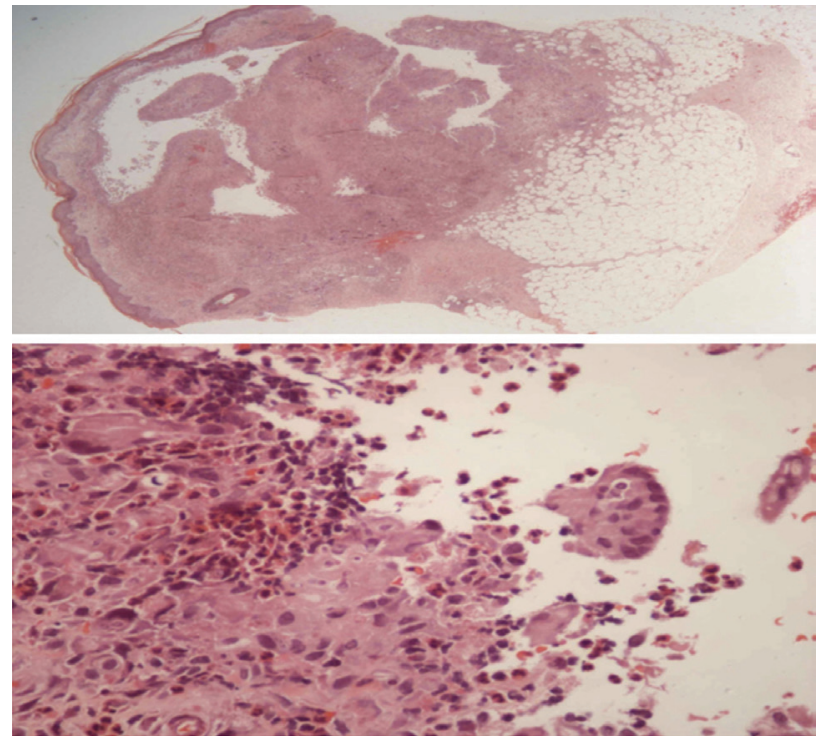

Figure 3 - Top image: intense mixed inflammatory infiltrate with suppuration in the dermis and the hypodermis. HE, OM: $x 40$ magnification; Bottom image: granulomatous and suppurative inflammation with epithelioid and giant multinucleated histiocytes and neutrophils in the dermis. HE, OM: $x 400$ magnification.

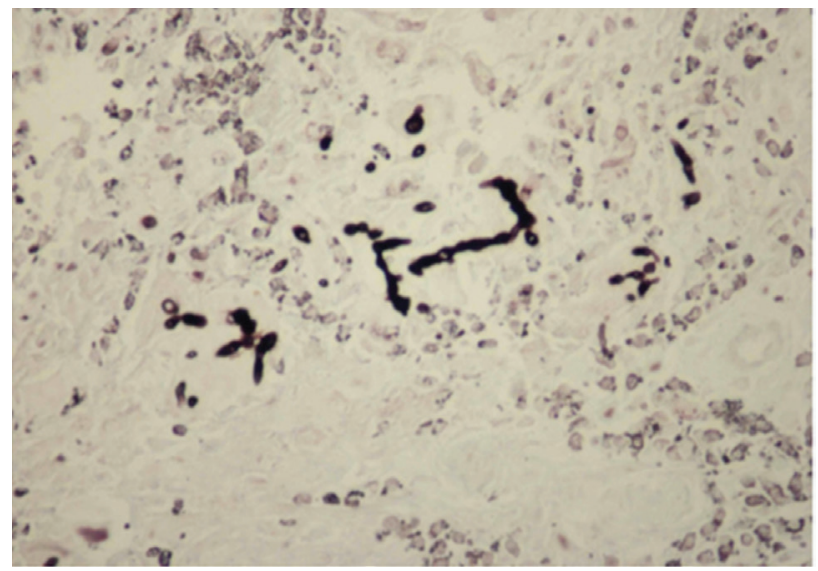

Figure 4 - Histopathology: Grocott's staining disclosing fungal hyphae in the dermis. OM: x 400 magnification. 


\section{DISCUSSION}

Dermatophytes generally cause cutaneous superficial mycosis by invading dead keratinized tissue, as dermatophytes are highly specialized parasites of keratinized layers of the skin, but under certain conditions, are capable of living and multiplying in the dermis and/or subcutis, as was observed in our patient ${ }^{6}$. Their hyphae penetrate the stratum corneum and hair and cause mechanical damage to the skin and hair followed by secondary inflammation and immune response. Furthermore, less commonly, the infection of dermis and hypodermis can occur as an extent of the disease in the presence of intense inflammation and rupture of the hair follicle, with subsequent granulomatous reaction seen in the histopathological analysis. Cell mediated immunity and delayed hypersensitivity reaction acting as a protective mechanism, contribute to the clinical picture of the dermatophyte infection. These lesions are mostly found on the lower limbs of female patients caused by Tricophyton rubrum ${ }^{3,5}$. Arthroderma gypseum (Nannizzia gypsea) is a geophilic fungus and its natural habitat is the soil. The diagnosis is usually based on history, clinical examination, direct microscopy, fungal culture and histopathological examination.

Invasive diseases due to dermatophytes are frequently associated with chronic superficial dermatophytosis as well as other factors such as the use of topical corticosteroids, atopic skin conditions and indwelling catheters ${ }^{7}$. Immunocompromised patients have an increased incidence of dermatophyte infections, are more prone to relapses and chronic infections, and are at risk for invasive disease $^{7}$. In transplant recipients on immunosuppressive medications, superficial dermatophyte infections are more common than the usual in immune compromised patients.

In fact, most of the reports described extensive lesions caused by different sorts of fungi ${ }^{8,9}$. Reviewing the current literature, we found only two clinical cases similar to our case, caused by Arthroderma gypseum $^{10,11}$ in immunosuppressed patients, making our clinical case unusual from a scientific point of view.

Furthermore, the most similar condition in comparison with ours is Wilson's granulomatous nodular perifolliculitis on the legs, considered a variation of Majocchi's granuloma, described by Wilson et al. ${ }^{12}$ in 1954 , but caused by T. rubrum. Moreover, there are three well-described forms of invasive dermatophyte infections: Majocchi's granuloma, a deeper dermal dermatophytosis, and a disseminated dermatophytosis ${ }^{7}$. The indolent form is known as Majocchi's granuloma, which may be subclassified into two types: (i) a fungal suppurative folliculitis, observed in healthy subjects due to hair follicular invasion, often in immunocompetent patients, and (ii) the second type of Majocchi's granuloma, often referred to as a nodular granulomatous perifolliculitis, that is a deeper lesion found in immunocompromised patients and usually exhibits a greater burden of fungal organisms in the lesion ${ }^{7}$. In the last type, patients can develop several lesions, as well described by Lestringant et al. ${ }^{13}$ that treated the patients with immunosuppressant drug therapies for a non-Hodking's lymphoma and they developed a sudden outbreak of more than 100 subcutaneous nodules without granulomatous reaction.

Follicular dermatophyte infections of the dermis in immunocompetent patients usually occur in women after shaving their legs in a backward movement and inoculating fungal elements into the dermis ${ }^{12}$. As in our clinical case, the deep dermal and subcutaneous nodular form in an immunosuppressed patient is characterized by three phases: the first one is similar to Tinea corporis infections, with erythematous scaly plaques and pruritic border, progressing to the second phase with bigger painful violaceous nodules tending to ulcerate and characterizing the third phase, called the degenerative phase $\mathrm{e}^{12,14}$.

These firm or floating nodules are more commonly described on the legs, but they can also appear on the scalp, hands etc. ${ }^{14}$. Such lesions often resemble a deep bacterial pyoderma. Other comorbidities such as malnutrition, leukemia, lymphomas or immunosuppressive therapies may also affect the action of polymorphonuclear cells and macrophages, that constitute the defense against dermophyte dermal invasion ${ }^{14}$. However, the fact that dermatophytes rarely penetrate below the epidermis when patients have very low CD4 T cell numbers, for example in HIV-positive individuals, and inhibition of immune responses through the development of an ineffective $\mathrm{TH} 2$ response in common chronic infections suggest that the survival of viable fungi in the dermis or lymphatics, or the dissemination through the bloodstream seldom occurs despite the underlying reduced immune capacity, as observed in our patient ${ }^{15}$. To prove this observation, in experimental murine infections, ablation of this T-cell pathway leads to chronic, extensive, but not internally disseminated infections ${ }^{16}$.

Uncommon invasive subacute or chronic dermatophyte infections were reported by Araviysky et al. ${ }^{17}$, who found a deep infiltration of skin and subcutaneous tissues, lesions of lymph nodes, bones and cartilages, viscera and central nervous system, and these authors claimed that the characteristic features of a deep generalized granulomatous trichophytosis should be considered. Allen et al.$^{18}$ described a 15-year old girl who developed a tinea corporis due to Microsporum audouinii associated with energy and defective lymphocyte transformation as a consequence 
of a deficiency of an uncharacterized plasma factor, who developed a rapidly progressive left hemiparesis, generalized hyperreflexia and virtually total body surface involvement with erythematous, scaly, crusted and fissured plaques, and she finally recovered after a total dose of $1,500 \mathrm{mg}$ of amphotericin B.

Considering the rising number of immunosuppressed patients due to medications, infections or malignancies, physicians should be aware of the presence of atypical infections caused by dermophytes that can mimic bacterial infections, especially in immunocompromised subjects. We highlight the need to adopt a low threshold to perform a biopsy of papules, nodules or plaques with prolonged evolution, which are resistant to therapy.

Deep dermatophyte infections are a challenge in dermatological practice, both for their insight in diagnosis and management. Early and proper diagnosis decreases the risk of the infection dissemination among immunosuppressed patients and the subsequent morbidities. The early onset of the treatment with the combination of topical azoles and oral antifungals need to be considered for the management of these patients.

\section{REFERENCES}

1. Oliveira WR, Tirico MC, Souza AA, Codarin FR, Silva LL, Festa Neto C. Skin lesions in organ transplant recipientes: a study of 177 consecutive brazilian patients. Int J Dermatol. 2019;58:440-8.

2. Imko-Walazuk BB, Predota A, Okuniewska A, Jaskiewicz J, Zegarska B, Placek W, et al. Superficial fungal infections in renal transplant recipients. Transplant Proc. 2014;46:2738-42.

3. Elevski BE, Sullivan J. Dermatophytes as opportunistic pathogens. J Am Acad Dermatol. 1994;30:1021-2.

4. Grossman ME, Pappert AS, Garzon MC, Silvers DN. Invasive Trichophyton rubrum infection in the immunocompromised host: report of three cases. J Am Acad Dermatol. 1995;33:315-8.

5. Novick NL, Tapia L, Bottone EJ. Invasive Trichophyton rubrum infection in the immunocompromised host. Am J Med. 1987;82:321-5.
6. Mayou SC, Calderon RA, Goodfellow A, Hay R. Deep (subcutaneous) dermatothyte infection presenting with unilateral lymphedema. Clin Exp Dermatol. 1987;12:358-88.

7. Marconi VC, Kradin R, Marty FM, Hospenthal DR, Kotton CN. Disseminated dermatophytosis in a patient with hereditary hemochromatosis and hepatic cirrhosis: case report and review of the literature. Med Mycol. 2010;48:518-27.

8. Bencini PL, Montagnino G, De Vecchi A, Tarantino A, Crosti C, Caputo R, et al. Cutaneous manifestations in renal transplant recipients. Nephron. 1983;34:79-83.

9. Shuttleworth D, Philpot CM, Salaman JR. Cutaneous fungal infection following renal transplantation: a case control study. Br J Dermatol. 1987;117:585-90.

10. Luscombe HA, Bingul O. Nodular granulomatous perifolliculitis caused by Microsporum gypseum. Arch Dermatol. 1964;89:274-6

11. Pereiro-Miguens M. Micosis granulomatosa por M. gypseum. Actas Dermosifiliogr. 1976;67:151-8.

12. Wilson JW, Plunkett OA, Gregerson A. Nodular Granulomatous perifolliculitis of the legs caused by Trichophyton rubrum. AMA Arch Derm Syphilol. 1954;69:258-77.

13. Lestringant GG, Lindley SK, Hillsdon-Smith J, Bouix G. Deep dermatophytosis to Trichophyton rubrum and T.verrucosum in an immunosuppressed patient. Int J Dermatol. 1988;27:707-9.

14. Das S, Saha R, Bhattacharrya SN. Disseminated nodular granulomatous perifolliculitis. Indian J Med Microbiol. 2007;25:288-90.

15. Hay RJ, Baran R. Deep dermatophytosis: rare infections or common, but unrecognized, complications of lymphatic spread? Curr Opin Infect Dis. 2004;17:77-9.

16. Calderon RA, Hay RJ. Cell-mediated immunity in experimental murine dermatophytosis. II. Adoptive transfer of immunity to dermatophyte infection by lymphoid cells from donors with acute or chronic infections. Immunology. 1984;53:465-72.

17. Araviysky AN, Araviysky RA, Eschkov GA. Deep generalized trichophytosis. Mycopathologia. 1975;56:47-65.

18. Allen DE, Snyderman R, Meadows L, Pinnell SR. Generalized Microsporum audouinii infection and depressed cellular immunity associated with a missing plasma factor required for lymphocyte blastogenesis. Am J Med. 1977;63:991-1000. 